\title{
HIDRÓLISE ÁCIDA PARA PRODUÇÃO DE NANO LIGNINA EM PÓ
}

\author{
ACID HYDROLYSIS FOR PRODUCTION OF NANO LIGNIN POWDER
}

\author{
Marina Stygar Lopes ${ }^{1}$, Mayara Elita Carneiro², Alan Sulato de Andrade, Daniele Cristina \\ Potulski \\ 1, 2, 3,4 Universidade Federal do Paraná, Curitiba, Paraná, Brasil-marinastygar@gmail.com, \\ mayaraecarneiro@gmail.com, alansulato@gmail.com \&danielepotulski@gmail.com
}

\begin{abstract}
RESUMO
O presente trabalho refere-se à produção e caracterização de nano lignina, ao utilizar uma hidrólise ácida em serragem, resíduo este gerado por serrarias. A nanotecnologia avança em diversos campos, devido ao seu grande potencial de novos usos para partículas em escala nanométrica. Consequentemente, o caráter renovável e biodegradável da lignina tem despertado interesse científico e econômico. A matéria prima deste estudo trata-se de serragem de Eucalyptus sp. Primeiramente, foi realizada a hidrólise ácida com ácido sulfúrico 72\% na proporção 2:1. O material foi homogeneizado durante duas horas e então conservado em temperatura ambiente. Posteriormente, visando produzir a nano lignina, este material foi processado no moinho Microprocessador Super Masscolloider Masuko Sangyo nas concentrações de 1 e $2 \%$. Para obter o pó da nano lignina foi necessário utilizar o equipamento denominado Spray Dryer, no qual o material é pulverizado dentro de uma câmara, terminando com a recuperação do material em pó. Estas nano ligninas em pó foram então caracterizadas pelas análises de espectroscopia de infravermelho médio, análise termogravimétrica, calorimetria exploratória diferencial, difração de Raios-x, microscopia eletrônica de transmissão e de varredura. As análises realizadas mostraram características intrínsecas de lignina nos dois materiais produzidos, o que comprova a eficiência da hidrólise ácida realizada ao obter lignina da serragem. A eficiência do uso do moinho, para produção de material em escala nano, foi afirmada pelas análises de caracterização microscópicas.
\end{abstract}

PALAVRAS-CHAVE: Eucalipto, Resíduo, Serragem, Tecnologia.

\begin{abstract}
The present work refers to the production and characterization of nano lignin, when using acid hydrolysis in sawdust, sawmills generate this residue. Nanotechnology is advancing in many fields because of its great potential for new uses for nanometer-scale particles. Consequently, the renewable and biodegradable nature of lignin has aroused scientific and economic interest. The raw material of this study is sawdust of Eucalyptus sp. Firstly, acid hydrolysis was performed with $72 \%$ sulfuric acid in a 2:1 ratio. The material was homogenized for two hours and then stored at room temperature. Subsequently, to produce nano lignin, this material was processed in the Super Masscolloider Masuko Sangyo Microprocessor mill at concentrations of 1 and $2 \%$. To obtain the nano lignin powder it was necessary to use the equipment called Spray Dryer, in which the material is sprayed into a chamber, ending with the recovery of the powder material. These nano powdered lignins were then characterized by analyzes of medium infrared spectroscopy, thermogravimetric analysis, differential scanning calorimetry, $\mathrm{x}$-ray diffraction, transmission, and scanning electron microscopy. The analyzes carried out showed intrinsic characteristics of the lignin in the two materials produced, which proves the efficiency of the acid hydrolysis carried out when obtaining lignin from sawdust. The efficiency of the mill's use for nanoscale material production was affirmed by microscopic characterization analyzes.
\end{abstract}

KEYWORDS: Eucalyptus, Waste, Sawdust, Technology. 


\section{INTRODUÇÃO}

A serraria é uma das principais indústrias florestais transformadoras da madeira bruta para a obtenção de madeira maciça. Os resíduos de madeira são resultantes de todas as fases do processo produtivo. Estes resíduos orgânicos constituem-se em fonte de impactos ambientais, pois produzem o chorume na sua decomposição, que ao entrar em contato com outros componentes tóxicos podem gerar uma grande quantidade de matéria orgânica, microrganismos, metais pesados e outros constituintes danosos para o meio ambiente (MARAGNO et al., 2007).

Atualmente, a queima e a compostagem são as alternativas praticadas para o tratamento de resíduos lignocelulósicos como a serragem, solucionando assim o problema do excesso de volumes dos resíduos biodegradáveis (BRANCO et al., 2005).

Porém, segundo Koch (2012), os resíduos oriundos da madeira podem ser tratados, reaproveitados e valorizados. Por isso é de fundamental importância que as indústrias estejam focadas em uma produção sustentável, pois para cada resíduo existem oportunidades de aproveitamento e lucratividade tecnicamente viáveis.

A utilização de materiais biodegradáveis, como a lignina, com potencial de reuso na cadeia produtiva, tem tornando-se realidade para muitas indústrias de transformação, como por exemplo, a adição de moléculas de filtros solares químicos aos filtros solares físicos (DAMASIO, 2015). Dentro deste contexto, as ligninas, consideradas os principais recursos renováveis aromáticos, representam uma excelente matéria-prima alternativa para a elaboração de produtos químicos e polímeros (LAURICHESSE \& AVÉROUS, 2014).

A extração da lignina de biomassa lignocelulósica representa o ponto chave para sua grande utilização em aplicações industriais. Embora atualmente seja usado como enchimento ou aditivo, a lignina raramente é explorada como matéria-prima para a produção química. No entanto, pode ser uma excelente candidata para modificações e reações químicas devido ao seu caráter altamente funcional, isto é, rica em grupos hidroxílicos fenólicos e alifáticos, para o desenvolvimento de novos materiais (LAURICHESSE \& AVÉROUS, 2014).

Aliado a isto, o motivo fundamental da maioria das aplicações da nanotecnologia é, justamente, a abundância de aplicações e possíveis variações nas propriedades dos materiais em escala nanométrica com a promessa de que os materiais terão funcionalidades melhoradas, justificando o interesse cada vez maior em estudos de materiais nanométricos (CHAUDHRY et al., 2010).
Existem diversos métodos para obtenção de materiais em escala nano. Dentre eles o método mecânico utiliza um moinho, que consiste em um disco rotatório e um disco fixo com uma abertura ajustável entre eles para que por meio do contato mecânico, o material possa ser reduzido a tamanhos menores (POTULSKI et al., 2016).

A modificação química da lignina conduziu inúmeros esforços e pesquisas com estudos significativos nas últimas décadas, como as pesquisas desenvolvidas por Gilca et al. (2014), que estudaram a obtenção de nano partículas de lignina a partir de modificação química, e Popa et al. (2011), que realizaram caracterizações de nanopartículas de lignina modificada quimicamente. Porém, estes trabalhos não visaram produzir e caracterizar a nano lignina que tenha sofrido o processo de secagem por atomização.

Dentro deste contexto, este trabalho teve por objetivo produzir e caracterizar nano lignina em pó, através do uso de uma hidrólise ácida na serragem de Eucalyptus sp.

\section{MATERIAL E MÉTODOS}

\section{Produção do material}

A amostra de serragem de Eucalyptus sp. foi fornecida em abril de 2016 pelo Laboratório de Usinagem, do DETF Setor de Ciências Agrárias da Universidade Federal do Paraná. Para a obtenção da lignina, a amostra sofreu uma hidrólise ácida com a adição de ácido sulfúrico $72 \%\left(\mathrm{H}_{2} \mathrm{SO}_{4}\right)$ na proporção 2:1, ou seja, $30 \mathrm{~mL}$ ácido para $15 \mathrm{~g}$ de serragem. O material homogeneizado foi conservado em temperatura ambiente.

Em um primeiro momento, para que o material fosse processado no moinho Microprocessador Super Masscolloider Masuko Sangyo (MKCA6-3; Masuko Sangyo Co., Ltd.), foi determinada a sua umidade, com base na massa seca, e a partir desta, calculada a massa de material e a quantidade de água necessária para o preparo de duas amostras com consistências de 1 e $2 \%$.

Os materiais seguiram então para o processo mecânico no moinho, utilizando oito passes e a frequência constante de $1.500 \mathrm{rpm}$. Os materiais, denominados 1 e $2 \%$, obtidos após o processamento no moinho, consistiam em um material aquoso, de textura fina e de cor marrom.

Os dois materiais produzidos no moinho foram destinados à secagem por atomização no Spray Dryer, no qual foram pulverizados individualmente dentro de uma câmara com parâmetros de processo controlados (vazão de $450 \mathrm{~mL} / \mathrm{min}$ e temperatura de $200^{\circ} \mathrm{C}$ ), terminando com a recuperação de nano ligninas em pó, de textura fina e 
coloração marrom escura.

\section{Caracterização do material}

Para a espectroscopia no infravermelho médio (FTIRMID) foram obtidos, para cada amostra, cinco espectros na região do infravermelho médio, na região espectral de 400 a $4.000 \mathrm{~cm}^{-1}$. 0 equipamento utilizado foi 0 espectrofotômetro Bruker Tensor 37 com 64 varreduras (scans) e resolução de $4 \mathrm{~cm}^{-1}$.

Para a análise termogravimétrica (TGA) e calorimetria exploratória diferencial (DSC), foi utilizado o equipamento Setaram Setsys Evolution TGA-DTA/DSC com cadinho de Alumina. Foi obtida uma leitura por amostra e as amostras foram aquecidas de 30 a $800^{\circ} \mathrm{C}$, a uma taxa de aquecimento de $20^{\circ} \mathrm{C} \cdot \mathrm{min}^{-1}$, em fluxo de argônio de 20 $\mathrm{mL} \cdot \mathrm{min}^{-1}$.

Para determinar a cristalinidade dos materiais, foi realizada a técnica de difração de Raios-x (DRX). O equipamento utilizado foi o difratômetro da marca Bruker, modelo D8 Advance. Foi realizada uma leitura por amostra e por intervalo. O ângulo de difração (2Ө), variou de 5 a $65^{\circ}$ e os intervalos utilizados foram de $0,02^{\circ}$ e $0,05^{\circ}$. Além disso, o equipamento foi operado a $40 \mathrm{kV}$ e corrente de 20 $\mathrm{mA}$. Este equipamento permite que a amostra gire em torno do seu eixo, enquanto as leituras são realizadas, fornecendo dados mais precisos. Além disso, os dados foram calculados diretamente pelo programa Difrac.eva versão 4.0 de 2014.

Para caracterização pela técnica de microscopia eletrônica de transmissão (MET), foi utilizado um Microscópio Eletrônico de Transmissão JEOL JEM 1200EXII, com resolução de 0,5 $\mathrm{nm}$. O material utilizado na análise foi preparado a partir da diluição de uma pequena quantidade do material em água destilada. Uma única gota do material em suspensão foi depositada sobre o portaamostra e, em seguida, seco à temperatura ambiente.

A técnica de microscopia eletrônica de varredura (MEV) foi realizada por meio do equipamento Microscópio de Varredura FEI Quanta 450 FEG, com resolução de $1 \mathrm{~nm}$. O material utilizado na análise foi preparado pela deposição de uma quantidade do pó sobre uma fita de cobre dupla face aderida ao porta-amostra.

\section{RESULTADOS E DISCUSSÃO}

\section{Caracterização por espectroscopia de infravermelho médio}

A seguir, os espectros obtidos para ambas as amostras de nano lignina (Figura 1).

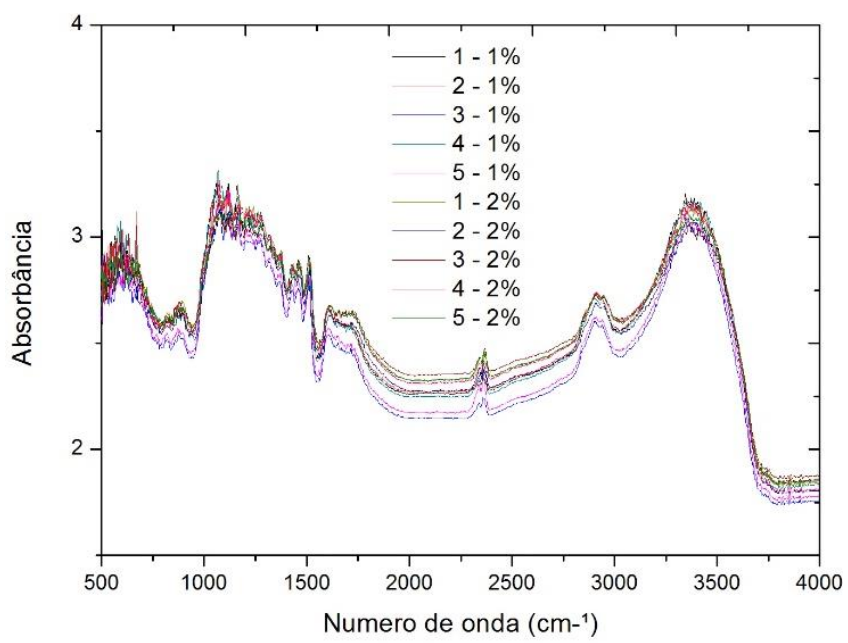

Figura 1. Espectros FTIR das amostras de nano lignina $1 \mathrm{e}$ $2 \%$.

É possível verificar bandas de absorção comuns para as duas amostras, pois ambas apresentaram os mesmos picos característicos.

Há absorção em $3.400 \mathrm{~cm}^{-1}$ atribuído aos grupos hidroxílicos $(\mathrm{OH})$, em $2.900 \mathrm{~cm}^{-1}$ à banda característica de $\mathrm{CH}$ alifáticos e entre 1.700 e $1.740 \mathrm{~cm}^{-1}$ referente ao alongamento $\mathrm{C}=\mathrm{O}$ característico de lignina e hemicelulose (DOBRICA et al., 2008).

$\mathrm{O}$ pico em $2.300 \mathrm{~cm}^{-1}$ está relacionado ao estiramento do grupo carboxílico $(C=0)$, sendo que a vibração molecular de $\mathrm{C}=\mathrm{O}$ nesta banda corresponde ao grupo das cetonas. Figueredo (2015), também observou estes resultados em seu trabalho com biocarvões de Eucalyptus.

Outro pico ocorre em $1.500 \mathrm{~cm}^{-1}$, referente à ligação $\mathrm{C}=\mathrm{C}$ do anel aromático da lignina. Travalini et al. (2016) observaram que esta banda característica em $1.500 \mathrm{~cm}^{-1}$, relacionando com as vibrações dos anéis aromáticos de lignina.

Também é possível observar bandas de absorção na faixa de $1.200 \mathrm{~cm}^{-1}$ e $1.300 \mathrm{~cm}^{-1}$, indicando a presença de anéis guaiacílicos e siringílicos respectivamente, próprio de folhosas como o Eucalyptus, na estrutura química das nano ligninas obtidas. Estes picos também foram observados por Saliba et al. (2001) e por Watkins et al. (2015) em estudos com ligninas.

$\mathrm{Em} 900 \mathrm{~cm}^{-1}$ ocorre um pico referente à vibração $\mathrm{C}$-H de celulose. Alekhina et al. (2015), também observaram este pico. Finalmente, observamos a ocorrência de um pico acentuado em aproximadamente $700 \mathrm{~cm}^{-1}$ característico da ligação $\mathrm{NH}_{2}$. Rodrigues et al. (2016) também destacam este pico de absorção em $702 \mathrm{~cm}^{-1}$.

Embora a técnica utilizada apresente somente um 
aspecto qualitativo, os resultados mostraram as características intrínsecas de lignina na estrutura química das nano ligninas produzidas.

\section{Caracterização por Análise Termogravimétrica}

A análise termogravimétrica é utilizada para verificar a estabilidade térmica e a temperatura de degradação, através das curvas de perda percentual de massa em função da temperatura. Segundo Watkins et al. (2015) as propriedades térmicas de ligninas podem apresentar diferenças, visto que dependem da sua origem.

A Figura 2 demonstra os estágios de degradação térmica para as amostras de nano lignina.

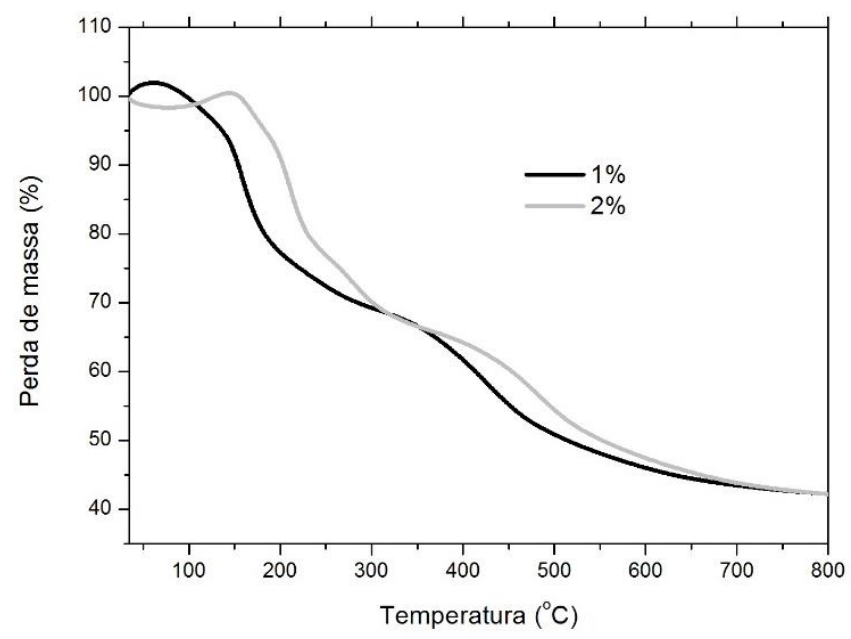

Figura 2. TGA das amostras de nano lignina 1 e $2 \%$.

Houve pequena perda de massa inicial até $120^{\circ} \mathrm{C}$, atribuída à perda de umidade das amostras. Trugilho et al. (2001) observou a ocorrência deste evento, em seu trabalho, relacionando-o com a secagem e consequente perda de umidade, caracterizando como uma reação endotérmica.

É possível observar um segundo evento de degradação térmica das amostras entre aproximadamente 160 e $350^{\circ} \mathrm{C}$, com perda de aproximadamente $30 \%$ de massa de ambas as amostras de nano lignina. A perda de massa nesta faixa de temperatura está relacionada com a degradação de carboidratos (WATKINS et al., 2015).

O terceiro evento de degradação ocorreu acima de $350^{\circ} \mathrm{C}$. Segundo Watkins et al. (2015), a degradação térmica nesta fase é devida aos produtos voláteis degradados derivados da lignina, incluindo fenólicos, álcoois, ácidos aldeídos, juntamente com a formação de produtos gasosos. É possível observar que as nano ligninas continuam se degradando, sendo que acima de $500^{\circ} \mathrm{C}$ ocorre a degradação dos anéis aromáticos.
Portanto, a lignina possui uma ampla faixa de degradação térmica. Segundo Brebu \& Vasile (2010), a ampla faixa de degradação, característica de lignina, está relacionada aos diferentes grupos funcionais oxigenados em sua estrutura, o que fornece diferentes estabilidades térmicas.

\section{Caracterização por calorimetria exploratória diferencial}

A análise de calorimetria exploratória diferencial é utilizada para verificar a taxa de perda de massa conforme o aumento de temperatura (Figura 3 ).

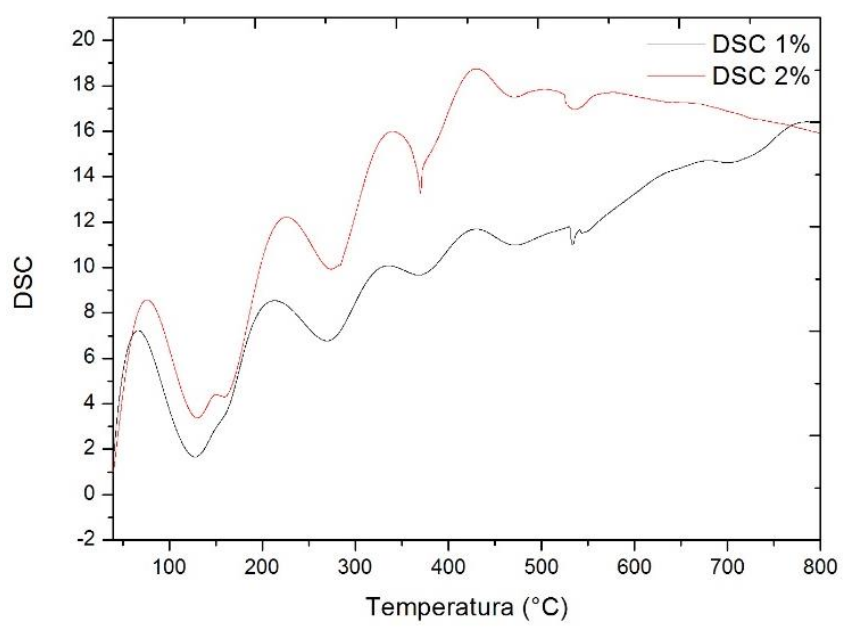

Figura 3. DSC das amostras de nano lignina 1 e $2 \%$.

As curvas de DSC obtidas para as nano ligninas apresentaram um pico endotérmico abaixo de aproximadamente $120^{\circ} \mathrm{C}$, estando este, relacionado ao calor necessário para evaporar a água das amostras. Apesar das amostras estarem secas no momento da análise, a total eliminação de água é dificultada. Travalini et al. (2016) indicaram a ocorrência deste evento em seus trabalhos, relacionando também com a evaporação da água.

Outro pico é observado próximo a $220^{\circ} \mathrm{C}$ estando relacionado com o início da degradação térmica da lignina. Liu et al. (2014), em seu trabalho com ligninas, observou picos em 225 e em $275^{\circ} \mathrm{C}$ referentes ao início da degradação de ligninas. Ainda, segundo Mousavion et al. (2012), nesta faixa de temperatura ocorre a ruptura das cadeias aromáticas.

O pico próximo a $340^{\circ} \mathrm{C}$ está relacionado à máxima degradação da lignina, com a quebra das ligações $\alpha$ - $\beta$-arilalquil-éter e quebra da ligação carbono-carbono, conforme já citado por (CARNEIRO, 2012). Resultado semelhante foi encontrado por Ibrahim et al. (2010) com degradação máxima da lignina em $352^{\circ} \mathrm{C}$. 
Houve também um pico em torno de $440^{\circ} \mathrm{C}$, que está relacionado aos processos de degradação das hemiceluloses residuais e da lignina (HOUREAU et al., 2004). Portanto, a análise de DSC complementa e verifica os resultados obtidos pela análise de espectroscopia no infravermelho para as nano ligninas.

\section{Caracterização por Difração de Raios-x}

Na Figura 4 observarmos os difratogramas para as amostras de nano lignina, denominadas 1 e $2 \%$.

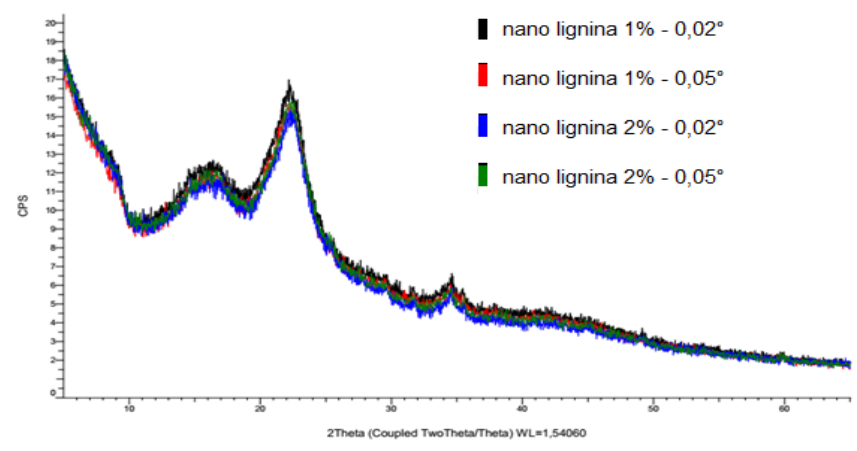

Figura 4. Difratograma de raios-x da nano lignina 1 e $2 \%$.

Para ambas as amostras e intervalos, verifica-se a presença de dois picos, sendo um de maior intensidade de $22^{\circ}$ e outro de menor intensidade de $16^{\circ}$ atribuídos à lignina amorfa. Resultados semelhantes aos obtidos para as nano ligninas são observados na literatura.

Goudarzi et al. (2014), ao realizarem análises de difração de Raios-x em lignina, encontraram um valor médio de pico de $21,5^{\circ}$. Kubo et al. (2003) relatou ângulo de difração de 22,7 graus para lignina de madeira dura. Já Ansari \& Gaikar (2013) relataram ângulo de $22,37^{\circ}$ para lignina.

Os valores de cristalinidade médios fornecidos pelo programa Difrac.eva 4.0 para a nano lignina $1 \%$ foi de $53 \%$ e para a nano lignina de $2 \%$ foi de $50,4 \%$. Neste trabalho, os índices de cristalinidade médios obtidos para ambas as nano ligninas são relativamente altos, considerando que a lignina é um material amorfo. A explicação para este valor de índice de cristalinidade, segundo Peng et al. (2012), está ligada ao uso da técnica de secagem por atomização em Spray Dryer.

Durante esta secagem os grupos hidroxilas disponíveis para interagir com a água provocaram aglomeração irreversível durante a retirada de água do sistema em elevada quantidade, ocorrendo a transformação do material líquido para o pó (ABE et al., 2007).

Além de ser considerada como uma técnica promissora, a secagem em Spray Dryer é, também, a que proporciona a obtenção de índices de cristalinidade mais elevados (PENG et al., 2013). Portanto, pode-se afirmar que o principal fator que contribuiu para os valores obtidos dos índices de cristalinidade foi a utilização da técnica de secagem por atomização em Spray Dryer.

\section{Caracterizações microscópicas}

As amostras de nano lignina $1 \%$ e $2 \%$, apresentaram as mesmas características para as análises de MET e MEV.

$\mathrm{Na}$ Figura 5, observam-se imagens obtidas por Microscopia Eletrônica de Transmissão.
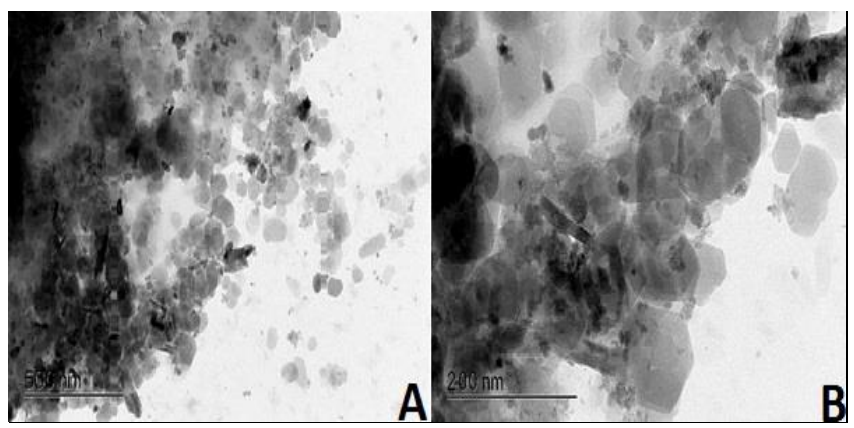

Figura 5. MET das amostras de nano lignina $1(A)$ e $2 \%(B)$.

Ao analisar estas imagens, observamos estruturas isoladas na escala nanométrica, algumas com menos de $200 \mathrm{~nm}$ de diâmetro. As estruturas se apresentam em aglomerados com variados formatos.

A análise em MEV evidencia a superfície do material a ser estudado. Nele, tem-se grande profundidade de foco, onde a morfologia fica evidente. As alterações morfológicas que ocorreram após a hidrólise ácida e a passagem do material pelo moinho são ilustradas na Figura 6.

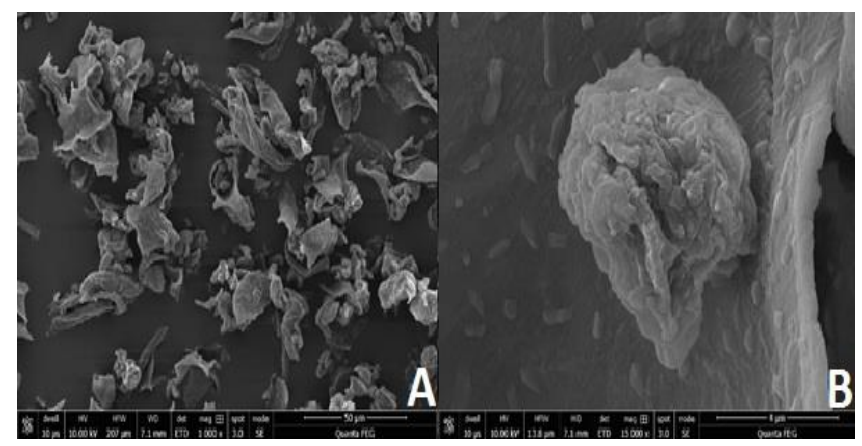

Figura 6. MEV das amostras de nano lignina 1 (A) e $2 \%$ (B).

Segundo Chaudhry et al. (2010), um material nano é definido como um material com uma ou mais dimensões externas em nano escala, ou seja, nano estruturado. Materiais que contenham as três dimensões em escala 
nanométrica são classificados como nanopartículas.

Ao analisar as imagens de MEV, observamos que os polímeros possuem morfologia irregular. O material se constitui de agregados compactos e sem formato definido, exatamente como a análise de MET demonstrou.

Andrade (2010), em seu estudo com lignina Kraft, observou grande heterogeneidade nas dimensões e formato das micropartículas de lignina Kraft obtidas. Estas apresentaram formatos difusos e com tamanhos que variam entre $1 \mu \mathrm{m}$ a $100 \mu \mathrm{m}$ em função do sistema de classificação adotado no estudo.

Portanto, as análises permitem confirmar a eficácia da hidrólise ácida realizada, pois não é possível observar estruturas características de celulose nos materiais produzidos. A eficácia no uso do moinho também é confirmada, pois é possível visualizar estruturas de lignina em escala nanométrica.

\section{CONCLUSÕES}

Por meio das diferentes análises de caracterização dos materiais produzidos, foi possível observar as características químicas e térmicas intrínsecas de lignina em ambas as amostras produzidas, ao apresentarem picos de absorção (FTIR-MID) e de degradação térmica (TGA e DSC) condizentes com a literatura para ligninas. Tais características observadas reforçam também a eficiência da hidrólise ácida realizada na serragem de Eucalyptus sp. para obtenção de lignina.

Ambas as análises de caracterização microscópica (MET e MEV) demonstraram que estruturas na escala nanométrica foram obtidas durante o processo mecânico. Além disso, o fato de ter-se obtido nano ligninas, comprova a eficiência do uso do moinho microprocessador e do número de passes determinado.

Por meio da análise de difração de Raios-x, ficou evidente que a secagem em Spray Dryer resultou em índices de cristalinidade elevados para lignina. Desta maneira, as nano ligninas em pó produzidas possuem características únicas que podem ser interessantes para diferentes usos industriais, mas principalmente para aquelas indústrias que utilizam este método de secagem durante o processo de produção.

\section{REFERÊNCIAS}

ABE, K.; IWAMOTO, S.; YANO, H. Obtaining cellulose nanofibers with a uniform width of $15 \mathrm{~nm}$ from wood. Biomacromolecules, v. 8, p. 3276-3278, 2007.

ALEKHINA, M.; ERSHOVA, O.; EBERTB, A.; HEIKKINENC, S.; SIXTA,
H. Softwood kraft lignin for value-added applications: Fractionation and structural characterization. Industrial Crops and Products, v. 66, p. 220-228, 2015.

ANDRADE, A. S. Utilização de micropartículas de lignina kraft combinadas com amido anfótero visando o aumento das propriedades do papel embalagem. 2010. $211 \mathrm{f}$. Tese (Doutorado em Engenharia Florestal) - Universidade Federal do Paraná, Curitiba, 2010.

ANSARI, K. B.; GAIKAR, V. G. Green hydrotropic extraction technology for delignification of sugarcane bagasse by using alkybenzene sulfonates as hydrotropes. Chemical Engineering Science, p. 157-166, 2013.

BRANCO, C. M.; BUDZIAK, C. R.; PAIXÃO, R. E.; MANGRICH, A. S. Aproveitamento de resíduo florestal para húmus. Revista da Madeira, n. 89, 2005.

BREBU, M.; VASILE, C. Thermal degradation lignin - a review. Cellulose chemistry and technology, v. 44, n. 9, p. 353-363, 2010.

CARNEIRO, M. E. Obtenção de nanosílica de equisetum arvenses I. e a sua utilização na modificação de lâminas de madeira de Schizolobium parahyba var. amazonicum (Huber ex Ducke) Barneby. 2012. 137 f. Tese (Doutorado em Engenharia Florestal) - Universidade Federal do Paraná, Curitiba, 2012.

CHAUDHRY, Q.; WATKINS, R.; CASTLE, L. Nanotechnologies in the food arena: new opportunities, new questions, new concerns. RSC Nanoscience \&Nanotechnology, v. 14, n. 14, p. 1-17, 2010.

DAMASIO, R. A. P. Caracterização e aplicações de celuloses nanofibrilada (CNF) e nanocristalina (CNC). 2015. $90 \mathrm{f}$. Dissertação (Mestrado em Ciência Florestal) - Universidade Federal de Viçosa, Viçosa, 2015.

DOBRICA, I.; BUGHEANU, P.; STANCULESCU, I.; PONTA, C. FTIR spectral data of wood used in Romanian traditional village constructions. In: Chimie Anul, 17., Anais..., Analele Universitatii din Bucuresti, v. 1, 2008, p. 33-37.

FIGUEREDO, N. A. Liberação de nutrientes e contaminantes e sorção de cádmio e chumbo por biocarvões derivados de fontes distintas. 2015. 45 f. Tese (Doutorado em Ciência e Tecnologia da Madeira) - Universidade Federal de Viçosa, Viçosa, 2015.

GILCA, I. A.; GHITESCU, R. E.; PUITEL, A. C.; POPA, V. I. Preparation of lignin nanoparticles by chemical modification. Iranian Polymer Journal, v. 23, p. 355-363, 2014.

GOUDARZI, A.; LIN, L. T.; KO, F. K. X-Ray diffraction analysis of kraft lignins and lignin-derived carbon nanofibers. Journal of Nanotechnology in Engineering and Medicine, v. 5, 2014.

HOAREAU, W.; TRINDADE, W. G.; SIEGMUND, B.; CASTELLAN, A.; FROLLINI, E. Sugarcane bagasse and caraua lignins oxidized chlorine dioxide and reacted with furfuryl alcohol: characterization and stability. Polymer Degradation and Stability, v. 86, p. 567-576, 2004.

IBRAHIM, M. M.; AGBLEVOR, F. A.; EL-ZAWAWY, W. K. Isolation and characterization of cellulose and lignin from steam-exploded lignocellulosic biomass. Bioresources, n. 5, v. 1, p. 397-418, 2010. 
$\mathrm{KOCH}, \mathrm{M}$. R. Gestão de resíduos sólidos de uma indústria de aglomerados e moveleira - um olhar para sustentabilidade. 2012. 126 f. Dissertação (Mestrado em ambiente e desenvolvimento) - Centro Universitário Univates, Lajeado, 2012.

KUBO, S.; YASUMITSU, U.; YOSHIHIRO, S. Catalytic graphitization of hardwood acetic acid lignin with nickel acetate. Journal of Wood Science, v. 49, n. 2, p. 188-192, 2003.

LAURICHESSE, S.; AVÉROUS, L. Chemical modification of lignins: towards biobased polymers. Progress in Polymer Science, v. 39, p. 1266-1290, 2014.

LIU, Y.; HU, T.; WU, Z.; ZENG, G.; HUANG, D.; SHEN, Y.; HE, X.; LAI, M.; HE, Y. Study on biodegradation process of lignin by FTIR and DSC. Environmental Science and Pollution Research, v. 21, p. 14004-14013, 2014.

MARAGNO, E. S.; TROMBIN, D. F.; VIANA, E. O uso da serragem no processo de minicompostagem. Engenharia Sanitária e Ambiental, v. 12, n. 4, p. 355-360, 2007.

MOUSAVIOUN, P.; GEORGE, G. A.; DOHERTY, W. O. S. Environmental degradation of lignin/poly (hydroxybutyrate) blends. Polymer Degradation and Stability, v. 97, p. 1114-1122, 2012.

PENG, Y.; HAN, Y.; GARDNER, D. J. Spray-drying cellulose nanofibrils: effect of drying process parameters on particle morphology and size distribution. Wood and Fiber Science, v. 44, n. 4, p. 448-461, 2012.

PENG, Y.; GARDNER, D. J.; HAN, Y.; KIZILTAS, A.; CAI, Z.; TSHABALALA, M. A. Influence of drying method on the material properties of nanocellulose I: thermostability and crystallinity. Cellulose, v. 20, n. 5, p. 2379-2392, 2013.

POPA, V. I.; CĂPRARU, A. M.; GRAMA, S.; MĂLUTAN, T. Nanoparticles based on modified lignins with biocide properties. Cellulose Chemistry and Technology, n. 45, v. 3-4, p. 221-226, 2011.

POTULSKI, D. C.; VIANA, L. C.; MUNIZ, G. I. B. de; ANDRADE, A. S. de; KLOCK, U. Caracterização de nanofilmes de celulose nanofibrilada obtida em diferentes consistências. Scientia Forestalis, v. 44, n. 110, p. 361-372, 2016.

RODRIGUES, V. C.; DINIZ, M. F.; MATTOS, E. C.; DUTRA, R. C. L. Separação e identificação de aditivos em tinta por TLC-IR/UATR e extração seletiva. Polímeros, v. 26, p. 68-74, 2016.

SALIBA, E. O. S.; RODRIGUEZ, N. M.; MORAIS, S. A. L. VELOSO, D. $P$. Ligninas - métodos de obtenção e caracterização química. Ciência Rural, v. 31, n. 5, p. 917-928, 2001.

TRAVAliNI, A. P.; PRESTES, E.; PINHEIRO, L. A.; DEMIATE, I. M. Nanocelulose de elevada cristalinidade extraída da fibra do bagaço de mandioca. O Papel, v. 77, n. 1, p. 73-80, 2016.

TRUGILHO, P. F.; LIMA, J. T.; MORI, F. A.; LINO, A. L. Avaliação de clones de Eucalyptus para a produção de carvão vegetal. Cerne, v. 7, n. 2, p. 104-114, 2001.
WATKINS, D.; NURUDDIN, M. D.; HOSUR, M.; NARTEH, A. T.; JEELANI, S. Extraction and characterization of lignin from different biomass resources. Journal of Materials Research and Technology, v. 4, n. 1, p. 26-32, 2015.

Recebido em 25/10/2017

Aceito em 12/12/2017 